\title{
AIMPORTÂNCIA DO MÉTODO: PESQUISA QUALITATIVA EM CONTEXTO DE SALA DE AULA
}

\author{
Gabriel Fortes Cavalcanti de Macedo ${ }^{1}$ \\ Nancy Lizeth Ramírez Roncancio² \\ Dayse Arianne Souza ${ }^{3}$
}

\begin{abstract}
RESUMO: Este artigo discute a importância de estudos naturalistas para observação de ambientes educacionais que visam aplicação prática do conhecimento acadêmico para sala de aula. A partir de três estudos realizados em um mesmo ambiente educacional, no qual a argumentação é sistematicamente praticada, se reflete sobre a importância da fundamentação metodológica e dos recursos analíticos na construção de um modelo qualitativo de ciência. Cada estudo é decorrente de uma pesquisa independente, com objetivos diferentes, os quais buscaram entender o impacto do desenho pedagógico nos processos psicológicos de alunos de um curso de Psicologia. A partir da reflexão se chama a atenção sobre as implicações práticas que advém da coerência entre o referencial epistemológico e as escolhas metodológicas e analíticas no contexto educativo e da aprendizagem, relação que garante a validez, confiabilidade e generalidade da pesquisa científica.

Palavras chave: Pesquisa Qualitativa. Ambiente Educacional. Desenvolvimento Cognitivo. Argumentação
\end{abstract}

THE IMPORTANCE OF THE METHOD: QUALITATIVE RESEARCH IN A CLASSROOM CONTEXT

ABSTRACT: This paper's goal is to discuss the importance of naturalistic studies to observe educational environments that aim classroom practical application of academic knowledge. From three studies done within the same argumentative educational setting, we discuss how methodological foundation and analytical resources gain relevance to understand how qualitative research models try to grasp the impact of a particular educational design and student's psychological processes. Each study discussed in this paper comes from independent researches that allow us to globally evaluate different aspects of the same classroom pedagogical design. Although they all come from the same theoretical background the three studies have different research goal making it possible to draw global and generalizable understanding of the impact of this pedagogical setting in the production, evaluation and the persistence of argumentative competences in different contexts.

Keywords: Qualitative research. Educational environment. Cognitive development.

\footnotetext{
1 Doutorando da Universidade Federal de Pernambuco - UFPE. E-mail: fortes-gabriel@hotmail.com

2 Doutoranda da Universidade Federal de Pernambuco - UFPE.

${ }^{3}$ Doutoranda da Universidade Federal de Pernambuco - UFPE.
} 
Argumentation.

\section{LA IMPORTANCIA DEL MÉTODO: INVESTIGACIÓN CUALITATIVA EN SALA DE CLASE}

RESUMEN: Este artículo discute la importancia de investigaciones naturalistas en ambientes educacionales que buscan la aplicación práctica del conocimiento académico en el aula. A partir de tres estudios realizados en un mismo ambiente educacional, en el que la argumentación es sistemáticamente practicada se reflete sobre la importancia de la fundamentación y de los estudios analíticos en la construcción de un modelo cualitativo de ciencia. Cada estudio es tomado de una investigación independientes, con objetivos diferentes, los cuales buscan entender el impacto de un diseño pedagógico en los procesos psicológicos de alumnos de un curso de Psicología. A partir de la reflexión se llama la atención sobre las implicaciones prácticas que resultan de la coherencia entre el referencial epistemológico y las elecciones metodológico/analíticas en contextos educativos y de aprendizaje, relación que garantiza la validez, confiabilidad y generalización de la pesquisa científica.

Palabras clave: Investigación Cualitativa. Ambiente Educativo. Desarrollo Cognitivo. Argumentación.

\section{Introdução}

Cada vez mais se discute a importância de construir cenários educacionais que proporcionem uma experiência formativa fundada em parâmetros científicos e que acompanhem o desenvolvimento da literatura educacional especializada. Assim, observa-se um aumento considerável de estudos que oferecem reflexão sobre aspectos metodológicos e/ou analíticos inovadores para a compreensão de fenômenos educacionais (LARRAín; FREIRE; HOWE, 2013; LEITÃO, 2011; MERCER, 2010). Contudo, poucos exploram a especificidade na operacionalização de estudos naturalistas, especialmente, quando se trata da generalização dos resultados sobre um mesmo ambiente observado (ASTERHAN; SCHWARZ, 2016; ERDURAN; OZDEM; PARK, 2015).

Este trabalho se propõe a refletir sobre as escolhas metodológicas que levaram à construção de três pesquisas sobre um mesmo ambiente educacional. Esse ambiente se caracteriza por ser construído em função de ciclos de debates regrados e as regras são explicitamente construídas a fim de que se cumpram as etapas da discussão crítica proposta pela escola pragma-dialética dos estudos de argumentação (VAN EEMEREN; GROOTENDORST; SNOECK HENKEMANS, 1996). A argumentação é, portanto, um modo de funcionamento para impulsionar a aprendizagem e também usada como artifício de desenvolvimento cognitivo dos estudantes (nas dimensões comunicacionais, cognitivas e metacognitivas).

Na literatura educacional, a construção desses ambientes vem sendo estudada sob a égide 
do termo "design" educacional e ao relacionar o conhecimento sobre argumentação e seu uso para a sala de aula se cunhou o termo "design argumentativo" para sala de aula (ANDRIESSEN; SCHWARZ, 2009). Essa prática vem sendo sistematicamente estudada (em especial nos Estados Unidos e Europa) como forma de compreender o papel no desenvolvimento do pensamento reflexivo dos estudantes ao participarem de atividades argumentativas de diversas formas: na construção de um vocabulário crítico (MERCER; LITTLETON, 2007); no desenvolvimento do pensamento reflexivo (KUHN; UDELL, 2003); nas discussões de evidências científicas (JIMÉNEZ-ALEXANDRE, 2007); e o papel da argumentação na sala de aula no ensino de ciências e temas sociocientíficos (JIMÉNEZ-ALEIXANDRE; ERDURAN, 2007).

O pensamento crítico é historicamente associado ao 'fazer' do professor e à sala de aula (ENNIS, 1996); e a argumentação sempre foi peça fundamental nesta perspectiva. No entanto, o foco do presente artigo é discutir e compreender de que forma as escolhas metodológicas, que visem uma abordagem qualitativa e naturalista dos fenômenos da sala de aula, podem ser articuladas ao se comparar diferentes estudos. Este artigo tem a relevância de propor uma reflexão metodológica ao comparar diferentes estudos sobre um mesmo espaço educacional em que a argumentação foi usada como forma de desenvolvimento de elementos cognitivos e discursivos para além da testagem de hipóteses e do acompanhamento estatístico. Oferece-se uma reflexão sobre como relacionar escolhas metodológicas, a construção dos dados, os resultados e o acompanhamento micro analítico (BRANCO, 1997) do desenvolvimento dos participantes alvo.

Com o propósito de contribuir com essa reflexão, este artigo aborda o papel das escolhas metodológicas de três estudos realizados pelos presentes autores. Essas pesquisas, apesar de estarem constituídas por objetivos diferentes, voltados a examinar a avaliação, a produção e a transferência de habilidades argumentativas, compartilham como alvo de análise um ambiente de aprendizagem em que a argumentação acontece de forma intensiva e sistemática. Portanto, trataremos da importância dos recursos metodológicos e analíticos elencados nos estudos bem como a natureza e clareza dos objetivos propostos. Esses aspectos são considerados relevantes para garantir critérios de validez, confiabilidade e generalidade próprios da pesquisa científica.

No que tange à base teórica, esses estudos assumem como princípios norteadores a gênese e o funcionamento do psiquismo em uma perspectiva dialógica (GROSSEN; SALAZAR ORVIG, 2011), a natureza constitutiva da linguagem nos processos cognitivos (VIGOTSKI, 2000) e o desenvolvimento da cognição atrelado ao contexto sócio-histórico (VIGOTSKI, 2000). Nesse sentido, ao assumir a 
argumentação como uma atividade linguístico-discursiva, considera-se também que esta teria um papel constitutivo nos processos cognitivos (LEITÃO, 2007, 2008).

A perspectiva dialógica adotada neste estudo representa a compreensão de que o psiquismo humano é permeado por um constante diálogo (com outros e consigo mesmo) e que os processos do pensamento estão altamente associados com os modos pelos quais os seres humanos operacionalizam esta dimensão inter e intrapessoalmente (VALSINER; ROSA, 2007). Sendo assim, a linguagem, ou melhor, o signo (em sua forma mais explícita o signo linguístico, mas não somente este) é tomado como o elo metodológico e fenomenológico que liga funções mentais e relações sociais, nos aproximando, portanto, das teorias sociogenéticas do desenvolvimento humano (VIGOTSKI, 2000; ZITTOUN, 2006). E por último, por acreditar na íntima relação entre signo (signo linguístico principalmente) e pensamento, os estudos aqui apresentados partem da ideia de que estudar formas discursivas de interação se constitui também como uma forma de estudo sobre processos cognitivos uma vez que discurso e cognição são dois processos de um mesmo sistema dinâmico de produção de sentidos (LEITÃO, 2007; VALSINER, 2006).

Com relação ao quadro metodológico, os estudos aqui citados foram realizados independentemente e tiveram os seguintes objetivos: estudo 1 - analisar possíveis transformações dos critérios de avaliação de argumentos usados por estudantes universitários ao examinarem a qualidade dos argumentos produzidos por seus pares; estudo 2 - investigar o desenvolvimento da habilidade de produção argumentativa; estudo 3 - investigar a eventual persistência de competências argumentativas adquiridas pelo uso intensivo e sistemático em sala de aula para outros contextos. Apesar dos objetivos distintos, vale ressaltar que todos eles partem de uma proposta metodológica de observação naturalista de estudos de caso e de amostragem intencionada 4 .

Ao querer observar o impacto de uma experiência pedagógica nas formas de pensamento dos alunos, o interesse deste artigo se centra no percurso desenvolvimental sobre a organização do pensamento (BRANCO; VALSINER, 1997). Esse aspecto delimita tanto o início do recorte (contextual e singular dos participantes) como outras dimensões da análise (temporalidade, aspectos cognitivos e microgenéticos do desenvolvimento - internalização/apropriação), as quais visam dar conta da cadeia

\footnotetext{
${ }^{4}$ Segundo Morse (1991), a amostra intencional se refere a uma escolha baseada em critérios definidos previamente pelo pesquisador e que estão atrelados aos objetivos da pesquisa.
} 
de transformações na organização do pensamento dos estudantes.

Para cumprir com o objetivo do artigo, inicialmente será discutido sobre a questão conceitual de metodologia qualitativa e suas vantagens em função do tipo de problema de pesquisa. Em seguida, reflete-se sobre a importância de um desenho metodológico claro para construção de conhecimento confiável, válido e generalizável no âmbito da ciência Psicológica e Educacional. E finalmente, enfatizase a importância do rigor científico da pesquisa qualitativa a partir de escolhas metodológicas.

\section{Reflexão teórico-metodológica sobre estudos em ambientes naturalistas}

A pesquisa qualitativa em Psicologia é um termo genérico utilizado para definir abordagens que não tomam como primado a quantificação estatística e o controle absoluto de variáveis. Pelo contrário, exploram fenômenos que ocorrem em situação contextual no tempo e no espaço (DENZIN; LINCOLN, 2005; GÜNTHER, 2006). Uma consequência imediata desses princípios é que o pesquisador que usa metodologias qualitativas não concentra esforços em medir, prever ou controlar seu objeto de estudo como estratégias para alcançar o rigor científico, senão observa a ocorrência de fenômenos em sua realidade ecológica (BRANCO, 1997). O que se propõe, então, é entender fenômenos psicológicos, justamente em ambientes espontâneos, desprovidos de controle experimental e que podem ser observados de forma completa; abordagens herdeiras de compreensões humanistas, holistas e contextuais, cujo fim é o entendimento da condição humana em seu ambiente natural em contraposição ao rigor do controle de variáveis dos métodos experimentais (WAGONER, 2015).

Mesmo que se reconheça a existência de críticas à falta de poder de generalização dos resultados obtidos nos estudos de corte mais qualitativo (STERNBERG, 2010), isto não implica, necessariamente, que este tipo de pesquisa não ofereça outras formas de interpretação e compreensão dos fenômenos, que possam ser tratadas como generalizáveis. Assim sendo, ressalta-se que a generalização nas pesquisas qualitativas é compreendida a partir dos dados que cada caso observado sistematicamente oferece; e se consolida com base na aplicabilidade do modelo analítico gerado em situações mais diferentes possíveis (VALSINER, 2006). Uma das principais contribuições a partir deste olhar qualitativo para a investigação de processos do pensamento e da aprendizagem é tentar estabelecer de forma criteriosa a relação das diferentes condições de emergência dos movimentos de transformação (desenvolvimento) dos processos psicológicos estudados (neste trabalho a cognição) (BRANCO; VALSINER, 1997).

A abordagem adotada nas pesquisas apresentadas acarreta na construção de um desenho 
metodológico primordialmente contextual e naturalista que acompanha a relação entre processos psicológicos como parte do cenário em que o sujeito está inserido. Compreensão esta que assume a relação interdependente entre ambiente e sujeito, na qual o contexto é parte da organização psicológica dos fenômenos observados (GONZÁLEZ REY, 1997). Ou seja, a situação de sala de aula não é uma variável que influencia o comportamento, mas sim um ecossistema com particularidades que necessita de escolhas metodológicas específicas para ser investigado.

Por se tratarem de estudos sobre desenvolvimento, os aspectos temporalidade, contexto, singularidade do sujeito observado, condições de emergência e transformação progressiva do fenômeno estudado são centrais para construção metodológica. Entender essa multiplicidade é o que permite acompanhar o desenvolvimento humano (VALSINER, 2006), relacionando passado, presente e futuro na linha de desenvolvimento (MERCER, 2008; VALSINER, 2013). Esse olhar minucioso sobre processos de mudança permite compreender as condições de emergência e transformação dos fenômenos argumentativos analisados, desde os momentos iniciais do processo até os momentos de estabilização na cadeia sistêmica de desenvolvimento em que os sujeitos estão inseridos (VALSINER; ROSA, 2007).

Ainda sobre contextualidade e singularidade, Valsiner e Branco (1997) chamam atenção para a constante tensão observada, como impulsor de desenvolvimento humano, a relação entre sujeito e o ambiente no qual está inserido. Portanto, o foco das pesquisas sobre desenvolvimento humano precisa levar em consideração que esse sujeito se encontra situado em um tempo e um espaço histórico-discursivo que o provê com ferramentas culturais (GILLESPIE; ZITTOUN, 2010) e específicas, que dão suporte à emergência da singularidade contextualizada (MARKOVÁ, 2006). Com esses aspectos teóricos em vista, ressalta-se que as informações apresentadas nas três pesquisas, analisadas neste artigo, se caracterizam pela abordagem qualitativa, nos moldes aqui descritos, em nível de: construção, análise e compreensão dos fenômenos do desenvolvimento humano.

\section{Ambiente educacional}

Os três experimentos aqui relatados se caracterizam por estudarem um ambiente naturalista de sala de aula, no qual se aplicou uma estratégia pedagógica com ênfase no uso da argumentação, de forma sistemática e intensiva, visando favorecer a construção de um cenário que potencializa a reflexividade dos estudantes (LEITÃO, 2012). Portanto, a referida proposta pedagógica buscou atingir metas tanto no nível da aprendizagem de conteúdos curriculares por meio da argumentação 
(argumentar para aprender) como do desenvolvimento cognitivo (aprender a argumentar).

A argumentação é compreendida como uma atividade discursivo-cognitiva que pode ser melhorada mediante práticas de ensino específicas em sala de aula (KUHN, 2005; LEITÃO, 2012). Entende-se, também, que aprender a argumentar ajuda no desenvolvimento do pensamento críticoreflexivo, uma vez que estimula práticas de ponderação de posições contrárias (KUHN et al., 2013; LEITÃO, 2007), revisão de perspectivas (LEITÃO, 2007) e uma atitude crítica frente à realidade (CHÁVEZ; FUENTES, 2011).

O cenário proposto por Leitão (2012) está organizado por meio da realização de ciclos de discussão crítica que seguem uma mesma sequência de fases e ações pedagógicas. A primeira fase, engajamento/familiarização, introduz o conteúdo curricular que fundamenta o tópico que será debatido. A segunda fase, preparo para o Debate Crítico, oferece explicitamente conteúdo conceitual e prático em argumentação - identificação, produção e avaliação de argumentos - para discutir a qualidade do conteúdo curricular. A terceira fase, realização do Debate Crítico, busca o uso intensivo e sistemático da dimensão cognitivo-discursiva de argumento, contra-argumento e resposta (LEITÃO, 2007), assim como a avaliação dos argumentos usando 'critérios normativos' de qualidade do argumento, aceitabilidade, relevância e suficiência (GOVIER, 2010). Nessa fase, a discussão crítica se realiza mediante etapas estruturadas de debate - restrito, aberto, encerramento. Finalmente, na quarta fase, fechamento, o professor encerra o ciclo esclarecendo as dúvidas que ainda persistem sobre o conteúdo.

Esse ambiente se caracteriza pela consideração sistemática entre pontos de vista antagônicos, ponderação da palavra alheia e antecipação de contra-argumentos. O desenho pedagógico estabelece modos de regulação do discurso em sala de aula (por meio da figura da professora e de estudantes de graduação e doutorado que desempenham o papel de monitores), no modo de organização das atividades dentro e fora da sala de aula, tomando como elemento estruturante a proposta com ações discursivas para uso da argumentação em sala de aula (LEITÃO, 2011), criando uma arquitetura específica para a construção de um "espaço pensante" (PERRETCLERMONT, 2015).

Não é propósito deste estudo a validação do modelo observado. Porém, vale informar que o modelo em questão se apropria do conhecimento pedagógico e de literatura internacional sobre estudos em ambiente de sala de aula no intuito de formatar as atividades e os ciclos propostos. A inserção de parâmetros dialógicos (ALEXANDER, 2008) vem sendo estudada como uma alternativa 
para a construção de uma sala de na qual a circulação de diferentes pontos de vista se torna um veículo para construção de um ambiente democrático, sugerindo maior autonomia dos estudantes em relação à discussão (HAYES; MATUSOV, 2005). Além disso, a argumentação como prática sistemática é vista como forma de beneficiar o pensamento crítico (KUHN; GOLDSTEIN; CROWEL, 2009), a metacognição (KUHN et al. 2013), o trabalho com temas sociocientíficos (JIMÉNEZ-ALEIXANDRE; ERDURAN, 2007) e a melhoria na qualidade da produção de argumentos (KUHN, 2005; LEITÃO, 2007).

É importante esclarecer que os três estudos exploram o impacto do desenho pedagógico aqui descrito, especificamente, sobre desenvolvimento de diferentes competências do pensamento dos estudantes envolvidos, mas o objetivo, o recorte analítico, os participantes e os indicadores analíticos correspondem às particularidades de cada pesquisa. Igualmente, se destaca que os objetivos pedagógicos não são subordinados aos objetivos científicos dos estudos aqui apresentados. Isso é, os objetivos de sala de aula são norteadores dos fins pedagógicos do professor e os objetivos de pesquisa são guia de observação dos dados naturalistas por parte dos pesquisadores. Portanto, a estruturação do ambiente da sala de aula, não foi criada ou controlada para atender aos desejos das pesquisas; pelo contrário, seus objetivos se construíram ao redor da proposta pedagógica já formatada.

Como o contexto observado é de aprendizes (em média 40 estudantes) de uma disciplina do primeiro semestre letivo do curso de Psicologia (primeira experiência na universidade com a formação profissional e acadêmica na área) a análise do desempenho não adota uma postura normativa sobre a argumentação; pelo contrário, foca na transformação progressiva de competências argumentativas a partir de parâmetros descritivos, como acontecem as mudanças e de que maneira o desenho pedagógico proposto ajuda neste processo. Os três estudos fazem uso do mesmo banco de dados (pertencente ao Núcleo de Pesquisa em Argumentação, da Universidade Federal de Pernambuco), o qual os presentes autores ajudaram a construir durante o percurso de suas pesquisas. Os participantes de cada estudo foram selecionados de acordo com critérios específicos, delimitados pelos seus respectivos pesquisadores, de modo a atender às demandas dos seus objetivos. Assim, as três observações investigativas foram realizadas com base no mesmo grupo de estudantes, porém, não necessariamente com os mesmos estudantes (ou seja, a turma coincide, mas os participantes variam em cada estudo exposto). A reflexão aqui trazida é sobre como as escolhas metodológicas permitem ao pesquisador observar a ecologia da sala de aula em seu contexto e, globalmente, comparar com outras observações feitas sobre o mesmo ambiente.

Cabe ressaltar que a participação na pesquisa foi voluntária, respeitando as condições éticas 
e legais estabelecidas pela resolução 466/12, do Comitê de Ética. Os dados analisados respeitam e garantem a total confidencialidade da identidade e a privacidade dos participantes, a confidencialidade da instituição e dos dados gerados no contexto das referidas pesquisas.

\section{Escolhas metodológicas na observação de ambientes de aprendizagem}

Apresentamos as escolhas metodológicas das três pesquisas destacando a coesão e a coerência entre objetivos e procedimento metodológico, distinguindo tanto o olhar desenvolvimental, quanto discursivo e dialógico. Assim sendo, a seguir se discute a estruturação das pesquisas a partir de duas categorias gerais: escolhas metodológicas e resultados, aspectos estes sobre os quais se chama a atenção para os elementos comuns e distintos entre os estudos; assim como se ressalta a sistematização e rigorosidade na construção metodológica de base qualitativa.

Como já foi mencionado durante o texto, por se tratar de pesquisas com objetivos diferentes, seus alvos de análise tangem noções argumentativas diferentes. Nesse sentido, na pesquisa desenvolvida por Ramírez (2012), o foco de atenção esteve direcionado sobre a avaliação de argumentos, noção que se refere ao exame objetivo, sistemático e contextualizado dos argumentos usados em defesa de um ponto de vista, assim como a ponderação da força argumentativa e da relação premissa-conclusão durante a argumentação. Na pesquisa realizada por Souza (2013), a noção foco de interesse foi a produção de argumentos, questão que pressupõe a relação dialógica entre posições discursivas que se desafiam de maneira organizada e fundamentada. Igualmente, assume que independente da função desempenhada numa discussão os produtores de argumentos organizam suas afirmações a partir de estruturas nas quais pontos de vista são fornecidos junto com razões (de diversas naturezas) que os fundamentam. Finalmente, na pesquisa realizada por Macêdo (2014), o interesse esteve voltado principalmente sobre os conceitos: desenvolvimento, entendido como os processos de mudança e estabilização de uma determinada forma de organização intra e intersubjetiva (VALSINER, 2006), e habilidades argumentativas - fundamentação do ponto de vista, antecipação de argumentos, contra-argumentos e operadores discursivos -, que foram usadas para incentivar a discussão e as tarefas do grupo de estudantes. 


\section{Escolha dos participantes e do contexto}

A amostra dos estudos aqui relatados foi selecionada de forma intencional, de acordo com critérios não aleatórios, pré-estabelecidos por seus respectivos pesquisadores (MORSE, 1991). As amostras foram selecionadas de uma turma regular de primeiro período, de um curso de Psicologia pertencente a uma Universidade Pública Brasileira. Essa turma (em média 40 estudantes) participou da implementação de uma experiência pedagógica baseada no uso sistemático e repetitivo da argumentação. Desse conjunto de estudantes, a partir dos objetivos e critérios específicos de cada pesquisa, foram escolhidos os casos para análises. Por exemplo, Ramírez (2012) privilegiou como critério de escolha alunos que tivessem participado uma ou mais vezes, nas posições de debatedor e juiz, resultando em sete casos para a análise. Souza (2013) privilegiou cinco casos/alunos que participaram, no mínimo duas vezes, das situações de produção argumentativa - debates e ensaios - e que participaram na posição de debatedor em pelo menos um dos debates realizados durante a intervenção. Por fim, Macêdo (2014) privilegiou três casos/alunos, os quais participaram do debate "atípico", situação que marcou o aparecimento de usos informais das regras formais do Debate Crítico.

A escolha de um número reduzido de participantes/casos, (sete - cinco - três, respectivamente), se justifica pela quantidade de dados a serem construídos e analisados no nível microanalítico, e pelo fato de que a generalização não depende da amostra populacional, mas sim da capacidade analítica de observar a densidade com que um fenômeno aparece. Igualmente, a metodologia de estudo de caso (YIN, 2011) foi escolhida por ser a mais apropriada para investigar os fenômenos em foco nos três estudos. Ao acompanharmos casos podemos inferir processos de mudança e estabilização em diferentes contextos garantindo um olhar qualitativo e naturalista, preservando a singularidade na investigação (VALSINER; ROSA, 2007).

A pesquisa com estudos de caso visa, em termos últimos, à generalização de um modelo teórico aplicado a casos múltiplos e distintos. Portanto, a generalização do conhecimento eventualmente gerado nessas pesquisas se consolidará não mediante uma replicação dos resultados para situações semelhantes, mas sim a partir da aplicabilidade do modelo gerado em situações mais diferentes possíveis (VALSINER, 2006).

Em consequência, os critérios de escolha estabelecidos em cada estudo proporcionaram um cenário heterogêneo para a construção do corpus da pesquisa (YIN, 2011), permitindo, assim, a observação de variações intra - sujeito e inter - sujeito nas diferentes situações escolhidas para a 
análise. Além disso, oferece variabilidade na hora de fazer as análises garantindo não só o exame de casos típicos, como também dos atípicos. Com isso, compreende-se que, no âmbito da pesquisa qualitativa, escolhemos uma amostra representativa não em termos estatísticos ou como representação da realidade de uma população básica, mas sim uma amostra capaz de representar a importância do fenômeno estudado (FLICK, 2009).

\section{Escolha do corpus}

Tendo em vista que estamos lidando com análise de mudanças concomitantes entre discurso e cognição, chamamos este procedimento metodológico de construção do corpus e não de dados (GONZÁLEZ REY, 1997). Assim sendo, o alvo de análise são as relações dialógicas no discurso dos estudantes em sala de aula no intuito de refletir sobre processos psicológicos e de desenvolvimento cognitivo. Essa é uma maneira de demarcar o lugar acadêmico de onde se comunica um estudo e de que maneira a análise e os resultados podem ser compreendidos (AARTS; BAUER, 2002).

Nesse sentido, o corpus de dados de uma pesquisa se refere a materiais naturais coletados (SINCLAIR, 1991), a partir de amostras estabelecidas intencionalmente pelo pesquisador, usadas para avaliar e entender a realidade em que os fenômenos são inseridos (GONZÁLEZ REY, 1997). A construção do corpus se iniciou com inspeção minuciosa das transcrições dos registros videográficos e dos materiais escritos produzidos pelos estudantes ao longo da disciplina, entre os quais foram selecionados aqueles que fariam parte do corpus (material a ser analisado) de cada estudo. Essa primeira escolha estabelece o possível recorte das situações dialógicas para a análise dos fenômenos tratados nos estudos aqui referidos.

Como se observa no Quadro 1, o corpus construído nas pesquisas apresentadas está inserido no contexto mais amplo do uso sistemático e intensivo da argumentação em sala de aula, mas pelas características e noções específicas próprias a cada pesquisa. Nesse caso, as habilidades argumentativas treinadas durante a proposta pedagógica - identificação de argumentos, produção de argumentos, critérios de avaliação, diferenças entre senso comum e senso crítico etc. -, são aspectos que circunscrevem e delimitam as situações e materiais privilegiados no corpus. Por exemplo, Ramírez (2012) escolheu situações e materiais ricos em movimentos de avaliação de argumentos; Souza (2013), situações e materiais ricos em movimentos de produção de argumentos; e Macêdo (2014), situações próprias do contexto inicial e outras situações nas quais as habilidades argumentativas poderiam emergir. 
Quadro 1 - Corpus de Análise

\begin{tabular}{|c|c|c|c|}
\hline $\begin{array}{c}\text { Critérios Avaliação de } \\
\text { Argumentos (RAMíREZ, 2012) }\end{array}$ & $\begin{array}{l}\text { Produção Argumentativa } \\
\text { (SOUZA, 2013) }\end{array}$ & $\begin{array}{c}\text { Persistência Habilidades } \\
\text { Argumentativas (MACEDO, } \\
\text { 2014) }\end{array}$ & \\
\hline $\begin{array}{l}\text { (a) Avaliação on-line. } \\
\text { Avaliações escritas } \\
\text { realizadas, pelos alunos, } \\
\text { durante os debates } \\
\text { ocorridos no transcurso da } \\
\text { disciplina. O foco do exame } \\
\text { foi a qualidade da } \\
\text { argumentação oferecida } \\
\text { pelos debatedores no dia do } \\
\text { debate. } \\
\text { (b) Avaliação pós-debates. } \\
\text { Momento de reflexão e } \\
\text { tomada de consciência por } \\
\text { parte dos alunos sobre } \\
\text { procedimentos e raciocínios } \\
\text { usados na própria prática do } \\
\text { debate. } \\
\text { (c) Coavaliação que os alunos } \\
\text { realizam dos ensaios } \\
\text { produzidos por seus pares. }\end{array}$ & $\begin{array}{l}\text { (a) Produção oral } \\
\text { durante os debates, } \\
\text { independente da função } \\
\text { desempenhada } \\
\text { (proponente ou oponente). } \\
\text { Nesta situação os } \\
\text { estudantes oferecem } \\
\text { argumentos, contra- } \\
\text { argumentos e respostas em } \\
\text { torno do tema de } \\
\text { discussão. } \\
\text { (b) Ensaios temáticos. Nesta } \\
\text { situação de produção todos } \\
\text { os alunos deviam elaborar, } \\
\text { individualmente, um } \\
\text { ensaio, com estrutura pré- } \\
\text { definida, que igualmente } \\
\text { serviria de base para se } \\
\text { preparar para discussão do } \\
\text { debate. }\end{array}$ & $\begin{array}{l}\text { a) Debate Crítico. Série de } \\
\text { debates planejados na } \\
\text { disciplina a partir do uso de } \\
\text { regras de comunicação } \\
\text { próprias ao MDC. } \\
\text { b) Debate "atípico". Tipo de } \\
\text { 'debate', iniciado } \\
\text { espontaneamente pelos } \\
\text { estudantes, fora do âmbito } \\
\text { e das regras do Debate } \\
\text { Crítico. } \\
\text { c) Outras disciplinas cujo } \\
\text { trabalho em sala de aula } \\
\text { não se centrava em práticas } \\
\text { argumentativas, e cujo foco } \\
\text { temático de discussão eram } \\
\text { conteúdos de Psicologia } \\
\text { Social. } \\
\text { d) Grupo focal. Situação de } \\
\text { aula criada especificamente } \\
\text { para fins do estudo, na qual } \\
\text { se discute: Benefícios e } \\
\text { malefícios da greve na } \\
\text { universidade. } \\
\text { e) Rede social. Materiais } \\
\text { espontaneamente postados } \\
\text { em rede social (Facebook), } \\
\text { e nos quais os alunos } \\
\text { acreditavam ter feito uso } \\
\text { da argumentação para se } \\
\text { posicionar sobre um tema. } \\
\text { Os trechos foram } \\
\text { fornecidos no momento de } \\
\text { realização do grupo focal. } \\
\text { Situação mais afastada do } \\
\text { contexto pedagógico criado } \\
\text { na DIP (debate } \\
\text { estruturado). }\end{array}$ & 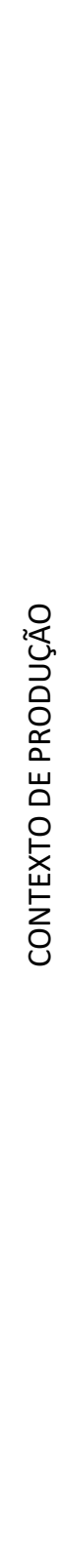 \\
\hline
\end{tabular}

Fonte: Ramírez (2012); Souza (2013) e Macêdo (2014).

\section{Indicadores analíticos}

São entendidos como parâmetros (quantitativo e/ou qualitativo) a partir dos quais se pode capturar e assinalar algumas tendências com relação aos fenômenos alvo de análise. Dentro desse plano teórico, destaca-se o conceito de 'unidade de análise', que se define como o menor recorte, ou 
seja, a 'menor' parte do fenômeno, na qual se preservam todas as suas propriedades básicas constituintes (VIGOTSKI, 2000). Dessa forma, por meio da unidade de análise é possível capturar as possíveis variações ou mudanças do fenômeno que está sendo investigado, de modo processual, em situações específicas.

A partir da escolha prévia de indicadores analíticos se observa que os pesquisadores buscaram preservar a observação da estrutura argumentativa em contextos naturais, de modo que utilizaram como parâmetro uma unidade de análise (como se vê no Quadro 2), capaz de capturar a dimensão dialógica da argumentação. No estabelecimento dos indicadores analíticos, os três estudos aqui apresentados compartilharam como elemento comum a Tríade Analítica de Leitão (2007) argumento, contra-argumento e resposta -, com a finalidade de identificar os episódios argumentativos; e no caso do estudo de Souza (2013), a tríade serviu, também, de base para a análise da estrutura básica da argumentação.

Quadro 2 - Indicadores Analíticos

\begin{tabular}{|c|c|c|c|}
\hline $\begin{array}{c}\text { Critérios Avaliação de } \\
\text { Argumentos (RAMíREZ, 2012) }\end{array}$ & $\begin{array}{l}\text { Produção Argumentativa } \\
\text { (SOUZA, 2013) }\end{array}$ & $\begin{array}{c}\text { Persistência Habilidades } \\
\text { Argumentativas (MACÊDO, } \\
\text { 2014) }\end{array}$ & \\
\hline $\begin{array}{l}\text { 1. Critérios Dialéticos ARS para a } \\
\text { avaliação da qualidade dos } \\
\text { argumentos (GOVIER, 2010): } \\
\text { Aceitabilidade, examina a } \\
\text { plausibilidade das justificativas. } \\
\text { Relevância, examina a relação } \\
\text { entre as justificativas e o ponto } \\
\text { de vista. Suficiência, examina se } \\
\text { o conjunto de justificativas } \\
\text { aporta bases adequadas e } \\
\text { suficientes para derivar delas a } \\
\text { conclusão. } \\
\text { 2. Critérios Pragmáticos. } \\
\text { Avaliam a qualidade da situação } \\
\text { discursiva que possibilita que a } \\
\text { argumentação seja criada e } \\
\text { sustentada. }\end{array}$ & $\begin{array}{l}\text { 1. Argumento (A), a ação de } \\
\text { oferecer um ponto de vista } \\
\text { justificado. Também se usou as } \\
\text { categorias de Walton, (1996) } \\
\text { para diferenciar os esquemas } \\
\text { argumentativos. } \\
\text { 2. Contra-Argumento (CA). } \\
\text { Ação de desafiar o ponto de } \\
\text { vista inicial. Também se usou } \\
\text { as categorias de Kuhn, } \\
\text { Goldstein e Crowell (2009), } \\
\text { para diferenciar CA com foco } \\
\text { no conteúdo desafiado. } \\
\text { 3. Resposta (R). Ação de } \\
\text { ponderar a relação entre A e } \\
\text { CA, em geral, adotando um } \\
\text { posicionamento diante da } \\
\text { questão. }\end{array}$ & $\begin{array}{l}\text { 1. Competências } \\
\text { explicitamente ensinadas: } \\
\text { Diferença entre opinião e } \\
\text { opinião fundamentada. } \\
\text { Estrutura argumentativa, } \\
\text { ponto de vista justificado, } \\
\text { contra-argumento e resposta } \\
\text { (LEITÃO, 2007). Tipos de } \\
\text { informação (objetiva, } \\
\text { subjetiva e hipotética). } \\
\text { Marcadores argumentativos e } \\
\text { suas funções. Critérios ARS } \\
\text { (GOVIER, 2010) para } \\
\text { avaliação da qualidade dos } \\
\text { argumentos. } \\
\text { 2. Competências discursivas } \\
\text { trabalhadas de forma } \\
\text { implícita: Dialogicidade. } \\
\text { Consideração da oposição. } \\
\text { Foco no desacordo. }\end{array}$ & 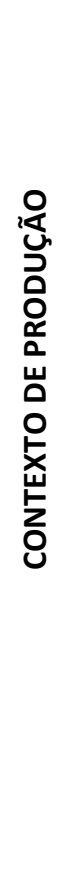 \\
\hline
\end{tabular}

Fonte: Ramírez (2012), Souza (2013) e Macêdo (2014). 


\section{Níveis de Análise}

Em razão da natureza dos fenômenos investigados e em coerência com os pressupostos teóricos assumidos, os estudos aqui descritos privilegiaram a análise discursiva e dialógica, para capturar os fenômenos em foco (produção, avaliação e persistência de habilidades). Nesse contexto, a investigação discursiva busca: 1) salientar como a linguagem constrói, regula e controla o conhecimento, as relações sociais e as instituições; 2 ) examinar as formas pelas quais os indivíduos utilizam ativamente a linguagem na construção de significados (AZEVEDO, 1998).

Com base nisso, os estudos descritos priorizaram três níveis de análise. O primeiro momento de produção de sentidos sobre o corpus analisado é a transcrição das filmagens (LEMKE, 1998). Nesse nível o pesquisador realiza uma revisão exaustiva das filmagens para obter uma compreensão holística do fenômeno investigado. No nível da microanálise, se realiza uma observação minuciosa e em detalhe dos indicadores analíticos estabelecidos, em especial, a observação de momentos de ruptura a partir dos quais se possa inferir processos de transformação e desenvolvimento (ZITTOUN, 2006). Finalmente, no nível da macroanálise há um acompanhamento global das mudanças ocorridas nos participantes ao longo do tempo. A partir da análise global é possível relacionar momentos de estabilidade quando se compara o momento inicial de investigação, a microanálise dos momentos de ruptura/transformação e, o ganho progressivo de estabilidade desse fenômeno em desenvolvimento.

A seguir apresentamos como exemplo um fragmento da análise realizada no estudo de Souza (2013) que ilustra o procedimento analítico. Para o caso específico, usaremos um fragmento referente ao debate regrado proposto para o ciclo 2, no qual estava em jogo a discussão sobre os "Pode-se afirmar que a Psicologia Diferencial adota uma perspectiva interacionista na explicação da constituição das diferenças individuais?". É importante também destacar que para esse ciclo os alunos já tinham sido trabalhados com os estudantes conteúdo da argumentação referente às diferenças entre opinião e opinião fundamentada, argumento, contra-argumento, resposta, argumento vs. explicação, marcadores discursivos, tipos de informação - sendo a informação objetiva a mais valorizada, uma vez que no contexto acadêmico é bastante pertinente que assim o seja e considerar as posições alternativas ao ponto de vista defendido (antecipar ou considerar a fala da bancada opositora.

O fragmento escolhido foi retirado da fase inicial do Debate (SOUZA, 2013) em que cada bancada poderia apresentar suas posições a partir dos argumentos construídos nas fases do ciclo, prévias ao dia de debate. Pertence a Tatiana (nome fictício) quem integrava o grupo responsável por 
defender o PV “A Psicologia Diferencial adota uma perspectiva interacionista na explicação da constituição das diferenças individuais". Postura que é defendida diante da justificativa "se observa que considera o inato como a pólvora [...] e o adquirido como gatilho [...]" (L 2-4), estabelecendo assim uma analogia entre inato/adquirido e pólvora/gatilho.

1 Pode-se afirmar que a psicologia diferencial toma sim uma postura interacionista a partir 2 do momento em que se observa que considera o inato como a pólvora, trazendo todas as 3 estruturas necessárias para desenvolver algumas características, e o adquirido como 4 gatilho, que vai trazer as condições para que a característica inata se desenvolva um 5 exemplo disto é, alguns indivíduos podem ter maiores chances de desenvolver transtornos 6 alimentares que outros, entretanto as condições ambientais podem ser determinantes para 7 que de fato um indivíduo apresente este distúrbio (Meyers, 2006).

Figura 1 - Fragmento retirado da fase inicial do Debate. Fonte: Dados do estudo.

Embora sua justificativa não contenha uma fonte explícita, a tese de que o ser humano está exposto à influência tanto de aspectos biológicos como do meio, está constituída de informação de natureza objetiva baseada nos textos-base dados como referência para a construção do ensaio. As linhas 5-7, fortalecem sua justificativa com um exemplo que ratifica a defesa do PV (a psicologia diferencial toma uma postura interacionista), apresentando explicitamente a fonte da informação (MEYERS, 2006). Portanto, a utilização de fonte confiável marca o apelo de Tatiana à informação objetiva na elaboração do argumento.

A partir da análise minuciosa dos argumentos produzidos pelos participantes escolhidos Souza (2013), pontua em que medida os aspectos enfatizados na proposta pedagógica da DIP (relativos à) se refletem no discurso produzido pelos participantes. De forma que, num nível macroanalítico, descreve as trajetórias de desenvolvimento da prática da produção de argumentos, capturáveis, somente, a partir de uma comparação da atividade argumentativa de cada participante escolhido em diferentes contextos. Assim sendo, a partir da microanálise oferecida se busca identificar as transformações qualitativas na habilidade de produção de argumentos dos casos analisados que permitem também identificar possíveis variações estruturais tendo em conta as diferentes trajetórias.

No caso do Quadro 3, se apresenta uma síntese geral dos resultados da macroanálise obtidos nas pesquisas referidas neste documento. 
Quadro 3 - Principais Resultados obtidos nos três estudos

\begin{tabular}{|c|c|c|c|}
\hline $\begin{array}{c}\text { Critérios Avaliação de } \\
\text { Argumentos (RAMÍREZ, 2012) }\end{array}$ & $\begin{array}{l}\text { Produção Argumentativa } \\
\text { (SOUZA, 2013) }\end{array}$ & $\begin{array}{c}\text { Persistência Habilidades } \\
\text { Argumentativas (MACEDO, } \\
\text { 2014) }\end{array}$ & \\
\hline $\begin{array}{l}\text { 1. Uso de Critérios dialéticos, } \\
\text { focado no exame minucioso da } \\
\text { estrutura interna dos } \\
\text { argumentos. } \\
\text { 2. Emergência de Critérios } \\
\text { pragmáticos que avaliam } \\
\text { aspectos da situação discursiva } \\
\text { que possibilitam que uma } \\
\text { argumentação seja criada e } \\
\text { sustentada. } \\
\text { 3. Transformações no uso de } \\
\text { critérios dialéticos e } \\
\text { pragmáticos. } \\
\text { 4. Todos os alunos focalizam } \\
\text { - em alguma medida - o uso } \\
\text { dos critérios, embora estes } \\
\text { não sejam igualmente } \\
\text { incorporados por todos. } \\
\text { 5. O ganho progressivo no } \\
\text { uso de critérios de avaliação, } \\
\text { marcado pelo aumento na } \\
\text { diversidade e densidade de } \\
\text { critérios que passam a utilizar. }\end{array}$ & $\begin{array}{l}\text { 1. Ganhos nas operações } \\
\text { argumentativas relativas à } \\
\text { estrutura dos argumentos. } \\
\text { 2. Apropriação da informação } \\
\text { objetiva como forma de } \\
\text { justificação dos pontos de } \\
\text { vista. } \\
\text { 3. Adensamento de esquemas } \\
\text { argumentativos relacionados } \\
\text { ao uso da figura de autoridade } \\
\text { como força argumentativa. } \\
\text { 4. Crescimento progressivo da } \\
\text { apropriação do modelo de } \\
\text { discurso argumentativo } \\
\text { ensinado. } \\
\text { 5. Ganhos relativos aos } \\
\text { esquemas argumentativos } \\
\text { relativos ao contexto de } \\
\text { produção e ao próprio } \\
\text { pensamento no campo } \\
\text { acadêmico. }\end{array}$ & $\begin{array}{l}\text { 1. Fundamentação objetiva de } \\
\text { opiniões (uso de fontes de } \\
\text { autoridade): persistência de } \\
\text { uso como recurso da produção } \\
\text { de argumento. Porém, a } \\
\text { formalidade ou informalidade } \\
\text { do contexto se mostra } \\
\text { determinante no que seria } \\
\text { escolhido como autoridade } \\
\text { para aquele contexto. } \\
\text { 2. Consideração de dois pontos } \\
\text { de vista antagônicos: } \\
\text { manutenção do parâmetro } \\
\text { dialógico nas discussões como } \\
\text { central para discussão formal } \\
\text { ou informal. } \\
\text { 3. Manutenção do foco na } \\
\text { avaliação da discordância } \\
\text { como forma de discussão de } \\
\text { um tópico antes que priorizar } \\
\text { o consenso. }\end{array}$ & 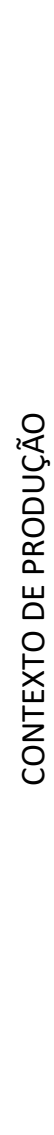 \\
\hline
\end{tabular}

Fonte: Ramírez (2012); Souza (2013) e Macêdo (2014).

\section{Generalizando o modelo e comparando diferentes estudos}

Levando em consideração o conjunto de informações oferecidas na sessão que trata sobre as escolhas metodológicas na observação de ambientes de aprendizagem, é possível agora pensar sobre a importância da comparação entre diferentes estudos como modo de generalização em estudos qualitativos. O elemento de comparação é interessante por tornar visível continuidades e discrepâncias entre os três estudos acerca de um mesmo ambiente educacional. Desse modo, refletiremos sobre os três estudos em termos "do que há de comum entre os resultados observados" e, não menos importante, "o que há de comum quanto ao que não foi observado".

No que diz respeito à similaridade entre os estudos, podemos notar três fatores importantes: ganhos na produção e avaliação de argumentos, maior presença de elementos dialógicos e 
pragmáticos na argumentação dos estudantes e apropriação do esquema argumentativo científico (especialmente focado na fonte de autoridade). Esses elementos podem ser vistos nos três estudos sob diferentes formas de observação e nos indica que a experiência pedagógica é produtiva no desenvolvimento dessas competências com os estudantes.

A produção e avaliação de argumentos diz respeito ao aumento progressivo do uso de justificativas para os pontos de vista produzidos pelos estudantes; além disso, o elemento de avaliação de argumentos passa de um plano subjetivo para incorporação de elementos críticos (e dialéticos) na forma como avaliam os argumentos dos colegas. A presença de elementos dialógicos e pragmáticos se refere à incorporação de elementos discursivos que sinalizam abertura para a discordância e consideração de diferentes vozes presentes na argumentação (cruciais para se estabelecer uma discussão produtiva em contexto acadêmico); e também de elementos de orientação da argumentação para atingir objetivos na discussão dentro e fora da sala de aula. Por último, a intensiva transformação (e persistência) do esquema argumentativo usado pelos estudantes nos indica apropriação dos "modos próprios de pensar e falar no campo científico", tendo em vista que os estudantes passam a integrar elementos de autoridade (fontes confiáveis) como forma de garantir a qualidade de seus argumentos.

Um fator "ausente" observado pode ser discutido. Os elementos dialéticos formais (ou de criticidade com os argumentos) se mostram menos impactantes do que elementos pragmáticos (foco na situação discursiva na qual está se argumentando). Isso pode ser explicado por alguns fatores: a não familiaridade com modelos formais de construção de argumentos; a forma intuitiva que na vida comum lidamos com produção e avaliação de argumentos; e, principalmente, porque no contexto de discussão em grupo, elementos formais podem dar lugar a formas socialmente compartilhadas de raciocinar e argumentar (MERCIER; SPERBER, 2011). No entanto, a apropriação de elementos próprios ao contexto científico indica que existem ganhos de criticidade quanto ao conteúdo discutido, especialmente, na preocupação com as fontes de autoridade usadas como elemento central dos argumentos.

\section{Conclusão}

Com base no exposto, se conclui que o recorte metodológico e as escolhas analíticas são cruciais para a construção de um modelo de ciência educacional que consiga dar conta do desempenho individual dos estudantes, promover diferentes modos de organização da cognição mediante impacto 
do ambiente social e garantir o alcance de certos objetivos pedagógicos. Apesar de se constatar na literatura um amplo conjunto de pesquisa concentradas no desenvolvimento de habilidades argumentativas em sala de aula, a maioria dos procedimentos empíricos adotados nesses estudos prioriza uma análise com ênfase no 'produto', e não nos 'processos' de desenvolvimento. Geralmente, esse tipo de pesquisa utiliza pré e pós teste para investigar desenvolvimento cognitivo (antes e depois de instruções específicas) e, portanto, se distancia de uma compreensão processual do diálogo (BAKER, 2015). Como consequência de tais escolhas metodológicas não se verifica em grande escala resultados que deem conta de explicar 'porquê' certas estratégias de ensino fracassam na promoção da aprendizagem, ou, em contrapartida, como mecanismos e/ou ferramentas presentes em designs pedagógicos específicos provocam construção do conhecimento, desenvolvimento de habilidades argumentativas e o pensamento crítico dos estudantes.

Uma vez que centramos nossas observações no aspecto discursivo e dialógico da atividade cognitiva, pudemos investigar profundamente a aprendizagem 'dentro e fora' da sala de aula. $O$ estudo da aprendizagem (e dos ambientes de aprendizagem), assim, deixam de ser o estudo dos resultados escolares para ser o estudo da prática social que permite compartilhar e construir conhecimento em situações específicas, com objetivos específicos, situados em um momento histórico no tempo. E em adição, este trabalho oferece a reflexão de como interpretar estudos qualitativos em seu contexto e em função das escolhas metodológicas feitas pelos pesquisadores, o que permite entender o modo de raciocínio que levou à construção dos dados e como se chegou nos resultados apresentados.

Essa marcação de que o estudo da cognição precisa de uma mirada dialógica vem sendo discutida por alguns autores (GROSSEN; SALAZAR ORVIG, 2011; ROMMETVEIT, 1992). Adotar tal perspectiva no intuito de compreender a ciência educacional requer discutir certos princípios metodológicos, a saber: observar a atividade real visando a validade ecológica e o adensamento da reflexão teórica em contrapartida à corrente de massificação dos dados que se vê hoje nas ciências humanas e sociais.

Nesse panorama, o presente artigo pretende contribuir com esse espaço de discussão, mostrando de que maneira é possível construir um percurso metodológico que possibilite a avaliação cuidadosa das práticas de intervenção em sala de aula. Os três estudos que apresentamos seguem preceitos teóricos e metodológicos parecidos; e a investigação a partir de um mesmo espaço educacional (cenário dos estudos descritos) que nos permite o aprofundamento da discussão acerca 
dos efeitos dessa intervenção sobre os modos de organização do pensamento dos participantes analisados.

Por fim, o que se pode afirmar é que os estudos discutidos neste artigo conseguiram capturar dimensões importantes para avaliar os ganhos no desenvolvimento cognitivo dos estudantes no nível da produção, da avaliação de argumentos e na permanência e transferência de algumas habilidades argumentativas em contextos diferentes daquele em que foram ensinados. Esses ganhos apenas puderam ser compreendidos em função das escolhas teóricas em conformidade com as escolhas metodológicas e analíticas.

\section{Referências}

AARTS, B.; BAUER, M. A. Construção do corpus: um princípio para a coleta de dados qualitativos. In: BAUER, M.; GASKELL, G. (Orgs.), Pesquisa qualitativa com texto, imagem e som: um manual prático. Petrópolis: Vozes, 2002. p. 39-63.

ALEXANDER, R. J. Culture, dialogue and learning: notes on an emerging pedagogy. In: MERCER, N.; HODGKINSON, S. (Eds.), Exploring Talk in School, 2008. p. 93-114.

ANDRIESSEN, J. E. B.; SCHWARZ, B. B. Argumentative design. In: A. N. PERRET-CLERMONT; MULLERMIRZA, N. (Eds.), Argumentation and education: theoretical foundations and practices. Dordrecht: Springer Verlag, 2009. p. 145-176.

ASTERHAN, C. S.; SCHWARZ, B. B. Argumentation for Learning: Well-Trodden Paths and Unexplored Territories. Educational Psychologist, v. 51, p. 164-187, 2016.

AZEVEDO, J. Metodologias qualitativas: análise do discurso. In: STEVES, A.; AZEVEDO, J. (Orgs.), Metodologias qualitativas para as ciências sociais. Porto: Instituto de Sociologia, 1998. p. 107-114.

BAKER, M. The integration of pragma-dialectics and collaborative learning research: dialogue, externalization and collective thinking. In: van EEMEREN, F. H.; GARSSEN, B. (Org.), Argumentation in Context. Amsterdam: John Benjamins Publishers, 2015, p. 175-199.

BRANCO, A. The "Ecological" Approach: When Labels Suggest Similarities beyond Shared Basic Concepts in Psychology. In: TUDGE, J.; SHANAHAN, M. J.; VALSINER, J. (Orgs.), Comparisons in Human Development Understanding Time and Context. Cambridge: Cambridge University Press, 1997. p. 304-317.

BRANCO, A.; VALSINER, J. Changing methodologies: A co-constructivist study of goal orientations in social interactions. Psychology \& Developing Societies, v. 9, n. 1, p. 35-64, 1997.

CHÁVEZ, P.; FUENTES, C. Nuevas Ciudadanías en Espacios Emergentes de Participación. Praxis. Revista de Psicología, n. 18, p. 65-89, 2010.

DENZIN, N. K.; LINCOLN, Y. S. Introduction: the discipline and practice of qualitative research. In: DENZIN, N. K.; LINCOLN, Y. S. (Orgs.), Handbook of qualitative research. Thousand Oaks: Sage Publications, 2005, p. 1-32. 
ERDURAN, S.; OZDEM, Y.; PARK, J. Y. Research trends on argumentation in science education a journal content analysis from 1998-2014. International Journal of STEM Education, v. 2, n. 5, p. 1-12, 2015.

ENNIS, R. H. Critical thinking. Upper Saddle River. NJ: Prentice-Hall, 1996.

FLICK, U. Qualidade na pesquisa Qualitativa. Porto Alegre: Artmed, 2009.

GILLESPIE, A.; ZITTOUN, T. Using resources: conceptualizing the mediation and reflective use of tools and signs. Culture \& Psychology, v. 16, n. 1, p. 37-62, 2010.

GONZÁLEZ REY, F. Epistemología cualitativa y subjetividad. La Habana, Cuba: Pueblo y Educación. 1997.

GOVIER, T. Good Arguments: An Introduction. In: GOVIER, T. (Org.), A Practical Study of Argument. Wadsworth: Cengage Learning, 2010. p. 87-115.

GROSSEN, M.; SALAZAR, O. A. Dialogism and dialogicality in the study of self. Culture \& Psychology, 2011, v. 17, n. 4, p. 491-509.

GÜNTHER, H. Pesquisa qualitativa versus pesquisa quantitativa: esta é a questão? Psicologia: Teoria e Pesquisa, Brasília, v. 22, n. 2, p. 201-209, 2006. Disponível em: <http://www.scielo.br/scielo.php?script=sci_arttext\&pid $=\$ 010237722006000200010 \& \operatorname{lng}=e n \& n r m=i s o>$. Acesso em: 22 nov. 2017.

HAYES, R.; MATUSOV, E. Designing for Dialogue in Place of Teacher Talk and Student Silence. Culture \& Psychology, v. 11, n. 3, p. 339-357, 2005.

JIMÉNEZ-ALEIXANDRE, M. P.; ERDURAN, S. Argumentation in Science Education: An Overview. In: ERDURAN, S.; JIMÉNEZ-ALEIXANDRE, M. P. (Orgs.), Argumentation in Science Education: Perspectives from Classroom-Based Research. Londom: Springer, 2007. p. 3-26.

KUHN, D. Enseñar a pensar. Buenos Aires - Madrid: Amorrortu Editores, 2005.

KUHN, D.; UDELL, W. The Development of Argument Skills. Child Development, v. 74, n. 5, p. 12451260. 2003.

KUHN, D.; GOLDSTEIN, M.; CROWELL, A. What Constitutes Skilled Argumentation and How Does it Develop? Informal Logic, v. 29, n. 4, p. 379-395, 2009.

KUHN, D. et al. Developing Norms of Argumentation: Metacognitive, Epistemological and Social Dimensions of Developing Argumentive Competence. Cognition and Instruction, v. 31, n. 4, p. 456496, 2013.

LARRAÍN, A.; FREIRE, P.; HOWE, C. Science Teaching and Argumentation: One-sided versus dialectical argumentation in Chilean middle-school science lessons. International Journal of Science, v. 36, n. 6, p. 1017-1036, 2014.

LEITÃO, S. Argumentação e desenvolvimento do pensamento reflexivo. Psicologia Reflexão e Critica, Porto Alegre, v. 20, n. 3, p. 454-462, 2007. Disponível em: <http://www.scielo.br/scielo.php?script=sci_arttext\&pid=S010279722007000300013\&Ing=en\&nrm=iso>. Acesso em: 22 nov. 2017.

LEITÃO, S. La dimensión epistémica de la argumentación. In: KRONMÜLLER, E.; CORNEJO, C. (Orgs.), Ciencias de la Mente: Aproximaciones desde Latinoamérica. Santiago, Chile: JC Sáez Editor, 2008. p. 89-119.

LEITÃO, S. O lugar de argumentação na construção do conhecimento. In: LEITÃO, S.; DAMIANOVIC, M. (Orgs.), Argumentação na escola: o conhecimento em construção. São Paulo: Pontes, 2011. p. 13-46. 
LEITÃO, S. O trabalho com argumentação em ambientes de ensino-aprendizagem: um desafio persistente. Uni-pluri/versidad (Medellín), v. 12, p. 23-37, 2012.

LEMKE, J. L. Analysing Verbal Data: Principles, Methods, and Problems. In: TOBIN, K.; FRASER, B. (Orgs.), International Handbook of Science Education. London: Kluwer Academic Publishers, 1998. p. 11751189.

MARKOVÁ, I. On "the inner alter" in dialogue. International Journal for Dialogical Science, v. 1, n. 1, p. 125-147, 2006.

MACÊDO, G. F. C. Desenvolvimento de habilidades argumentativas: do debate crítico à argumentação cotidiana. 2014. 102f. Dissertação (Mestrado em Psicologia Cognitiva) - Universidade Federal de Pernambuco, Recife, 2014.

MERCER, N. The seeds of time: Why classroom dialogue needs a temporal analysis. The Journal of the Learning Sciences, v. 17, n. 1, p. 33-59, 2008.

MERCER, N. The analysis of classroom talk: Methods and methodologies. British Journal of Educational Psychology, n. 80(Pt 1), p. 1-14, 2010.

MERCER, N.; LITTLETON, K. Dialogue and the development of children's thinking: A sociocultural approach. London: Routledge, 2007.

MERCIER, H.; SPERBER, D. Why do humans reason? Arguments for an argumentative theory. Behavioral and Brain Sciences, v. 34, n. 2, p. 57-74, 2010.

MORSE, J. Approaches to Qualitative-Quantitative Methodological Triangulation. Nursing Research, v. 40, n. 2, p. 120-123, 1991.

PERRET-CLERMONT, A-N. The Architecture of Social Relationships and Thinking Spaces for Growth. In: PSALTIS, C.; GILLESPIE, A.; PERRET-CLERMONT, A-N. (Orgs.), Social Relations in Human and Societal Development. Basingstokes (Hampshire, UK): Palgrave Macmillan, 2015. p. 51-70.

RAMÍREZ, N. L. R. Desenvolvimento do pensamento reflexivo: Um estudo de transformações na qualidade da argumentação de participantes do debate crítico. 2012. 193f. Dissertação (Mestrado em Psicologia Cognitiva) - Universidade Federal de Pernambuco, Recife, 2012.

ROMMETVEIT, R. Outlines of dialogically based social-cognitive approach to human cognition and communication. In: WOLD, A. H. (Org.), The dialogical alternative: Towards theories of language and minds. Oslo: Scandinavian University Press, 1992. p. 19-44.

SINCLAIR, J. Corpus, concordance, collocation. Oxford: Oxford University Press, 1991.

SOUZA, D. A. Aprender a argumentar: um estudo do desenvolvimento da produção. 2013. 141f. Dissertação (Mestrado em Psicologia Cognitiva) - Universidade Federal de Pernambuco, Recife, 2013.

STERNBERG, R. Introdução à Psicologia Cognitiva. In: STERNBERG, R. (Org.), Psicologia Cognitiva. Tradução M. LUCHE e R. GALMAN. São Paulo: Cengage Learning, 2010. p. 1-28.

VAN EEMEREN, F. H.; GROOTENDORST, R.; SNOECK HENKEMANS, F. Fundamentals of Argumentation Theory a Handbook of Historical Backgrounds and Contemporary Developments. Mahwah, NJ: Lawrence Erlbaum Associates, 1996.

VALSINER, J. The development of the concept of development: Historical and epistemological perspectives. In: LERNER, R. M. (Org.), Theoretical models of human development. Vol. 1 of Handbook of Child Psychology. 6. ed. Hoboken, NJ: Wiley, 2006. p. 516-568. 
VALSINER, J.; ROSA, A. The Cambridge Handbook of Sociocultural Psychology. Cambridge: Cambridge University Press, 2007.

VALSINER, J. Making of the future: Temporality and the Constructive Nature of Human Development. TURKEWITZ, G.; DEVENNY, D. A. (Orgs.), Developmental time and timing. Psychology Press, 2013, p. $13-40$

VIGOTSKI, L. S. A construção do pensamento e da linguagem. São Paulo: Martins Fontes. 2000.

WAGONER, B. Qualitative Experiments in Psychology: The Case of Frederic Bartlett's Methodology. Forum Qualitative Sozialforschung / Forum: Qualitative Social Research, [S.I.], v. 16, n. 3, 2015. Disponível em: <http://www.qualitativeresearch.net/index.php/fqs/article/view-/2367>. Acesso em: 22 nov. 2017.

WALTON, D. Argumentation schemes for presumptive reasoning. Hillsdale, NJ: Lawrence Erlbaum, 1996.

YIN, R. Choices in Designing Qualitative Research Studies. In: YIN, R. (Org.), Qualitative Research from Start to Finish. New York: The Guilford Press, 2011. p. 75-102.

ZITTOUN, T. Transitions: Development through symbolic resources. Greenwich, CT: Information Age, 2006. 